\title{
Hexadecadienoic Acid
}

National Cancer Institute

\section{Source}

National Cancer Institute. Hexadecadienoic Acid. NCI Thesaurus. Code C68372.

A polyunsaturated long-chain fatty acid with a 16-carbon backbone and exactly two double bonds. 